\title{
Wavelet-Based Approach for Structural Damage Detection
}

\author{
By Z. Hou, ${ }^{1}$ M. Noori, ${ }^{2}$ and R. St. Amand ${ }^{3}$
}

\begin{abstract}
A wavelet-based approach is proposed for structural damage detection and health monitoring. Characteristics of representative vibration signals under the wavelet transformation are examined. The methodology is then applied to simulation data generated from a simple structural model subjected to a harmonic excitation. The model consists of multiple breakable springs, some of which may suffer irreversible damage when the response exceeds a threshold value or the number of cycles of motion is accumulated beyond their fatigue life. In cases of either abrupt or accumulative damages, occurrence of damage and the moment when it occurs can be clearly determined in the details of the wavelet decomposition of these data. Similar results are observed for the real acceleration data of the seismic response recorded on the roof of a building during the 1971 San Fernando earthquake. Effects of noise intensity and damage severity are investigated and presented by a detectability map. Results show the great promise of the wavelet approach for damage detection and structural health monitoring.
\end{abstract}

\section{INTRODUCTION}

Damages are often observed in many engineering systems during their service life such as those in critical flight components in a helicopter, including cracks in a drive shaft (Champagne et al. 1998) and spalling of sun gear of a main rotor transmission (Samuel et al. 1998). Those damages may be caused by various factors such as excessive response, accumulative crack growth, and impact by a foreign object. Future intelligent structures demand high system performance, structural safety and integrity, and low maintenance cost. To meet the challenge, structural health monitoring (SHM) has emerged as a reliable, efficient, and economical approach to monitor system performance, detect damages if they occur, assess/diagnose the structural health condition, and make corresponding maintenance decisions.

An ideal SHM system typically consists of two major components: a built-in network of sensors for collecting response measurements, and a data analysis algorithm/software for interpretation of the measurements in terms of the physical condition of the structures. Recent flourishing developments in the area may be attributed to two factors: (1) innovative concepts such as structural controls and intelligent structures; and (2) advanced sensing techniques. The former requires an on-line health monitoring system to measure and diagnose the system performance data in a real-time manner. If a system fault or damage is detected, or the system performance becomes unsatisfactory, an appropriate control action can then be taken. The latter, such as development of the embedded distributed piezoelectric sensor arrays (Blanas et al. 1997) and embedded fiber optic sensors (Kim et al. 1993) in composite structures, may provide rich system performance data and make the online monitoring much more feasible. For recent advances in SHM, see Chang (1997).

A comprehensive literature review of damage detection and health monitoring methods for structural and mechanical systems was provided by Doebling et al. (1996). Their review focused on methods based on vibration measurements and de-

\footnotetext{
${ }^{1}$ Assoc. Prof., Mech. Engrg. Dept., Worcester Polytechnic Inst., Worcester, MA 01609.

${ }^{2}$ Prof. and Head, Dept. of Mech. and Aerosp. Engrg., North Carolina State Univ., Raleigh, NC 27695-7910.

${ }^{3}$ Formerly, Grad. Student, Mech. Engrg. Dept., Worcester Polytechnic Inst., Worcester, MA.

Note. Special Editor: Roger Ghanem. Discussion open until December 1, 2000. To extend the closing date one month, a written request must be filed with the ASCE Manager of Journals. The manuscript for this paper was submitted for review and possible publication on February 25, 2000. This paper is part of the Journal of Engineering Mechanics, Vol. 126, No. 7, July, 2000. (C)ASCE, ISSN 0733-9399/00/0007-0677-0683/ $\$ 8.00+\$ .50$ per page. Paper No. 22245 .
}

tection based on changes in vibration characteristics such as natural frequencies, modal shapes, and modal damping ratios. Intuitively, changes in these characteristics reflect variations in some physical parameters and working conditions of the system and indicate certain damages such as those caused by failure of joints and columns in a structure. Representative methods include "classical" system identification of natural frequencies and modal shapes; neural network approaches for predicting system performance by training (Wu et al. 1992; Tsou and Shen 1994); fuzzy logic and probability-based estimation to consider uncertainties (Vanik and Beck 1997; Essaway et al. 1998); and methods of localized flexibility for determination of damage location (Reigh and Park 1997).

This paper addresses wavelet analysis and its applications for structural damage detection. Wavelet analysis may be viewed as an extension of the traditional Fourier transform with adjustable window location and size. The merits of wavelet analysis lie in its ability to examine local data with a "zoom lens having an adjustable focus" to provide multiple levels of details and approximations of the original signal. Therefore, transient behavior of the data can be retained. Recent developments in mathematical theory of wavelets and their applications may be found in Chui (1992), Sone and Yamamoto (1997), and Benedetto and Frazier (1993).

Wavelet analysis has recently emerged as a promising tool for SHM and damage detection. Cumulative damage of a building with bilinear restoring force subjected to a real earthquake ground motion was estimated in terms of the accumulated ductility ratio, which is related to the number of spikes in the wavelet results (Masuda et al. 1995; Sone et al. 1995). The wavelet approach for on-line detection of an abrupt stiffness loss was studied and the results were compared with other approaches such as a neural network based on-line approximation technique and the empirical mode decomposition method (Demetriou and Hou 1999; Hou and Noori 1999; Vincent et al. 1999). Faults in gear systems were detected using wavelet approaches and some results were verified by an inspection (Wang and McFadden 1995; Staszewski and Tomlinson 1997; Ferlez and Lang 1998; Samuel et al. 1998). The operating condition for a silicon-wafer slicer cutting process was monitored and a maintenance decision of whether to sharpen the cutting blade could be made based on the wavelet analysis (Jiang et al. 1997). Effects of noise contamination and damage severity on damage detection were discussed (AlKhalidy et al. 1997a,b; Hou and Noori 1999). Most recent studies include Gurley and Kareem (1999) and Yamamoto et al. (unpublished manuscript, 1999). The latter used a vibratory gyroscope sensor to measure the jerk of a structure response, and the wavelet results were used to detect initiation and growth of a crack in an underground pipe. 
This paper is an extended version of the writers' previous paper (Hou and Noori 1999). In the following sections, a brief background of wavelet analysis is presented and characteristics of representative vibration signals under the wavelet transform are illustrated. The methodology is then applied to simulation data generated from a simple structural model subjected to a harmonic excitation. The model consists of multiple breakable springs, some of which may suffer irreversible damage when the response exceeds a threshold value or the number of cycles of motion is accumulated beyond their fatigue life. In cases of either abrupt or accumulative damages, occurrence of damage and the moment when the damage occurs can be clearly determined in the details of the wavelet decomposition of these data. Similar results are observed for the real acceleration data of the seismic response recorded on the roof of a building during the 1971 San Fernando earthquake. Effects of noise intensity and damage severity are investigated and presented by a detectability map.

\section{BACKGROUND OF WAVELET ANALYSIS}

This section presents a brief background on wavelet analysis utilized in this paper. General overview of wavelet analysis may be found in Chui (1992), Daubechies et al. (1992), and Sone and Yamamoto (1997).

Using a selected analyzing or mother wavelet function $\Psi(t)$, the continuous wavelet transform of a signal $f(t)$ is defined as

$$
(W f)(a, b)=\frac{1}{\sqrt{a}} \int_{-\infty}^{+\infty} f(t) \bar{\Psi}\left(\frac{t-b}{a}\right) d t
$$

where $a$ and $b=$ dilation and translation parameters, respectively. Both are real numbers and $a$ must be positive. The bar over $\Psi(t)$ indicates its complex conjugate. The mother wavelet should satisfy an admissibility condition to ensure existence of the inverse wavelet transform such as

$$
C_{\Psi}=\int_{-\infty}^{+\infty} \frac{\left|F_{\Psi}(\omega)\right|^{2}}{|\omega|} d \omega<\omega
$$

where $F_{\Psi}(\omega)$ denotes the Fourier transform of $\psi(t)$. The signal $f(t)$ may be recovered or reconstructed by an inverse wavelet transform of $(W f)(a, b)$ as defined by

$$
f(t)=\frac{1}{C_{\psi}} \int_{-\infty}^{+\infty} \int_{-\infty}^{+\infty}(W f)(a, b) \Psi\left(\frac{t-b}{a}\right) \frac{1}{a^{2}} d a d b
$$

A wavelet family associated with the mother wavelet $\Psi(t)$ is generated by two operations: dilation and translation. The translation parameter, $b$, indicates the location of the moving wavelet window in the wavelet transform. Shifting the wavelet window along the time axis implies examining the signal in the neighborhood of the current window location. Therefore, information in the time domain will still remain, in contrast to the Fourier transform, where the time domain information becomes almost invisible after the integration over the entire time domain. The dilation parameter, $a$, indicates the width of the wavelet window. A smaller value of $a$ implies a higherresolution filter, i.e., the signal is examined through a narrower wavelet window in a smaller scale.

In practical signal processing a discrete version of wavelet transform is often employed by discretizing the dilation parameter $a$ and the translation parameter $b$. In general, the procedure becomes much more efficient if dyadic values of $a$ and $b$ are used, i.e.,

$$
a=2^{j} ; \quad b=2^{j} k \quad j, k \in Z
$$

where $Z=$ set of positive integers. For some special choices of $\psi(t)$, the corresponding discretized wavelets $\left\{\psi_{j, k}\right\}$ where

$$
\Psi_{j, k}(t)=2^{j / 2} \Psi\left(2^{j} t-k\right)
$$

constitute an orthonormal basis for $L^{2}(R)$. Using the orthonormal basis, the wavelet expansion of a function $f(t)$ and the coefficients of the wavelet expansion are defined as

$$
f(t)=\sum_{j} \sum_{k} \alpha_{j, k} \Psi_{j, k}(t)
$$

and

$$
\alpha_{j, k}=\int_{-\infty}^{+\infty} f(t) \bar{\Psi}_{j, k}(t) d t
$$

In the discrete wavelet analysis, a signal can be represented by its approximations and details. The detail at level $j$ is defined as

$$
D_{j}=\sum_{k \in Z} a_{j, k} \Psi_{j, k}(t)
$$

and the approximation at level $J$ is defined as

$$
A_{J}=\sum_{j>J} D_{j}
$$

It becomes obvious that

$$
A_{J-1}=A_{J}+D_{J}
$$

and

$$
f(t)=A_{J}+\sum_{j \leq J} D_{j}
$$

Eqs. (10) and (11) provide a tree structure of a signal and also a reconstruction procedure of the original signal. By selecting different dyadic scales, a signal can be broken down into many low-resolution components, referred to as the wavelet decomposition tree. The wavelet tree structure with details and approximations at various levels may reveal valuable information of the signal characteristics that may not be clearly seen in the original data or the results from other approaches. A down-sampling technique (Strang and Nguyen 1996) can be used to efficiently reduce the data size in the tree and the Mallat algorithm (Mallat 1988), a fast wavelet transform procedure, can be used to greatly reduce the computational efforts involved. The analysis may be modified to the wavelet packet analysis for the purpose of multiresolution analysis, which provides an alternative tree structure for the original data. Higher dimensional versions can also be easily extended. For details, see Chui (1992).

\section{SOME RESULTS FOR REPRESENTATIVE VIBRATION SIGNALS}

This section presents results of wavelet analysis for some representative signals in vibration to bring insights into characteristics of vibration signals related to damage detection. The Daubechies wavelet DB4 was used in both continuous and discrete wavelet transforms.

Fig. 1 illustrates a unit-step signal, as shown in Fig. 1(a), and the results from its continuous and discrete wavelet transforms. Fig. 1(b) is the contour plot of the wavelet coefficient in the time-scale plane. Lighter shading in the plot indicates a higher wavelet coefficient value. The fringe pattern near the 450th time step implies existence of a singularity in the local time. The singularity is also indicated by a spike near that time step in Fig. 1(c). Considering that structural damage may be represented by an abrupt stiffness loss, the sensitivity of wavelet results to a singularity can be effectively used to detect possible structural damage using measured acceleration response data.

Fig. 2 presents the results of wavelet analysis for a sinu- 


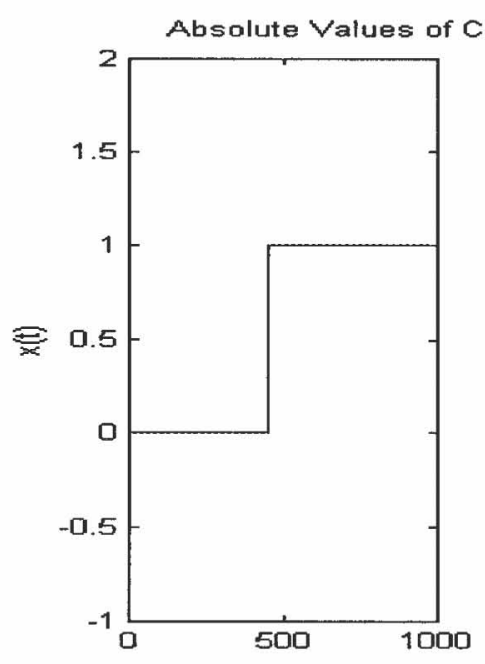

(a)

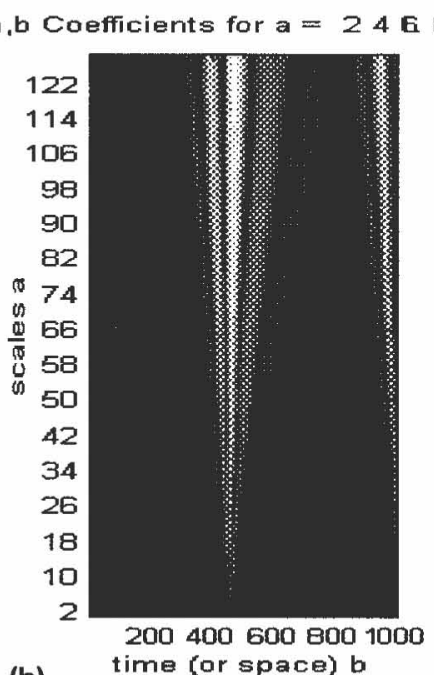

(b)

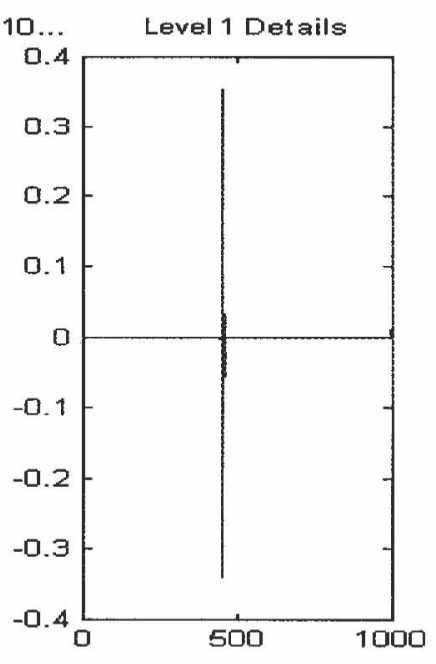

(c)

FIG. 1. Unit Step Signal and Results from Its Continuous and Discrete Wavelet Transforms: (a) Unit-Step Signal; (b) Its Continuous Wavelet Coefficient; (c) Details at Level 1 from Its Discrete Wavelet Transform

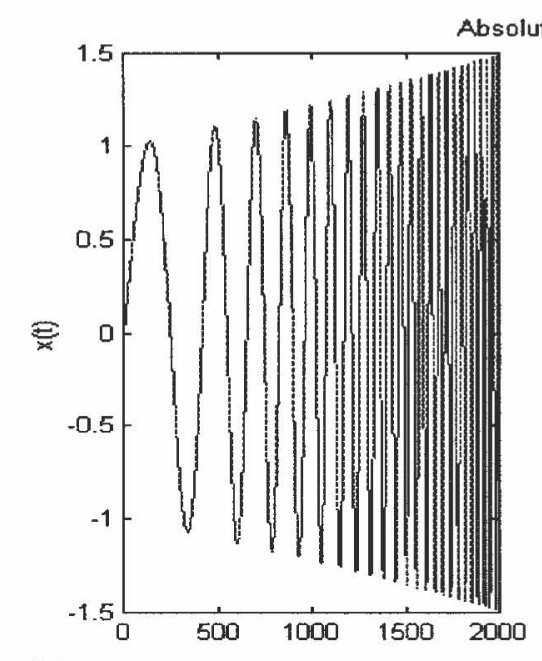

(a)

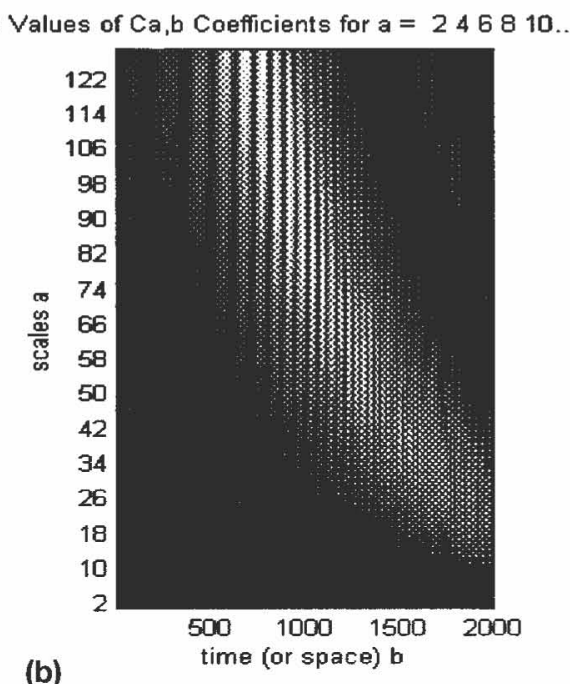

(b)

FIG. 2. Sinusoidal Signal with Sweeping Frequency and Its Continuous Wavelet Coefficients: (a) Sweeping Signal; (b) Its Continuous Wavelet Transform

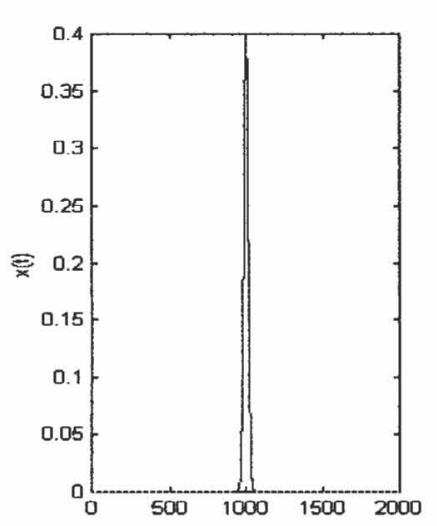

(a)

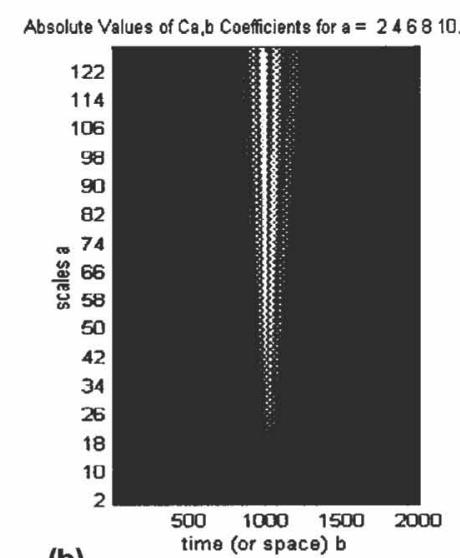

(b)

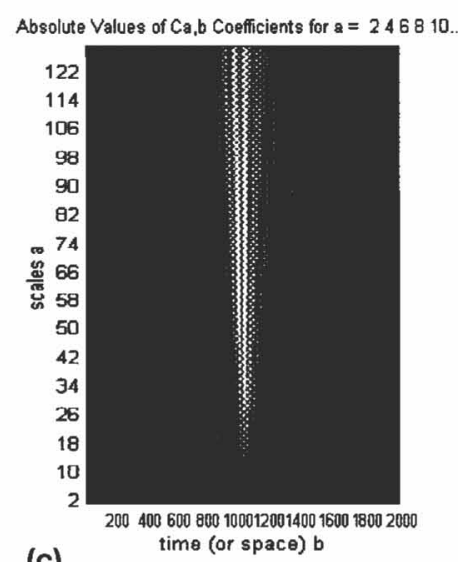

(c) ;; (b) Wavelet Coefficient of Signal; (c) Wavelet Coefficients of First-Order Derivative of Signal 


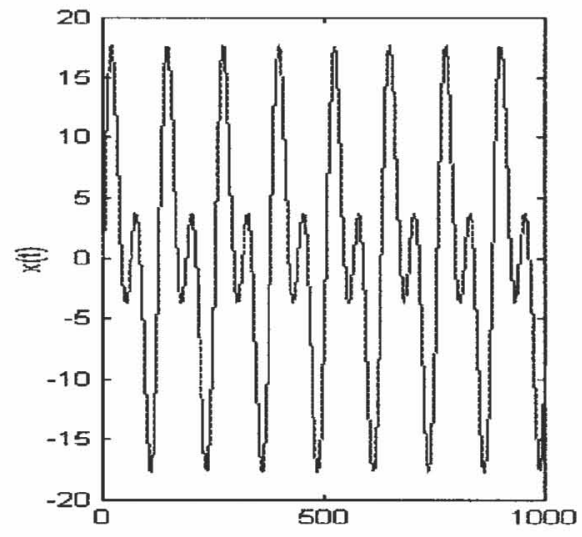

(a)

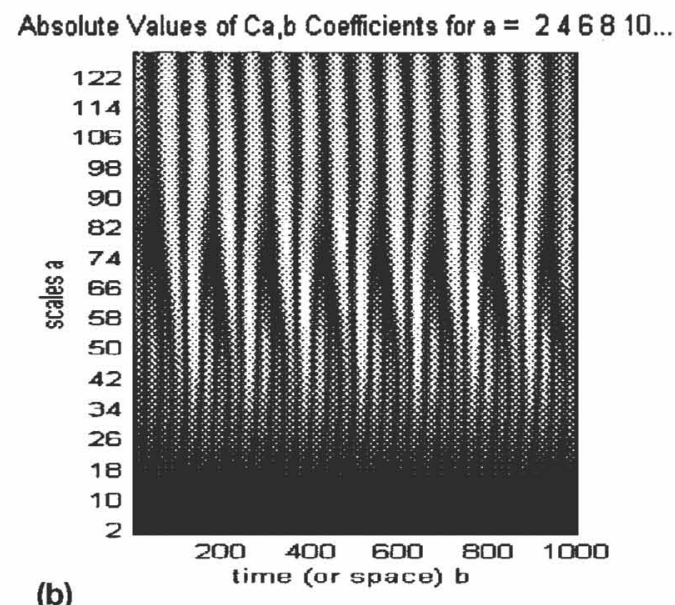

FIG. 4. Free-Vibration Signal and Its Continuous Wavelet Coefficient: (a) Original Signal; (b) Continuous Wavelet Coefficient

soidal signal with sweeping frequency. The results clearly show an increase in the transient frequency of the signal with time as indicated by a decreasing width of the fringe pattern in the contour plot of the wavelet coefficient, as shown in Fig. 2(b). The change in width of the fringe pattern implies that the dominant frequency in the signal increases with the time. The results bring an insight into detecting evolutionary or accumulative system damage caused by many factors such as mechanical fatigue and chemical corrosion. In this case, structural stiffness degradation may be indicated by increasing fringe width of the wavelet results with time for a vibration measurement from the structure.

Fig. 3 plots a localized and smooth signal as well as the wavelet coefficients of the signal itself and its first-order derivative. It is observed that the corresponding wavelet coefficients are also localized. The wavelet coefficients of higherorder derivatives exhibit a similar behavior. The plot may bring some insights to identify location or region of a damage, if occurred.

Fig. 4 demonstrates the continuous wavelet coefficient for a free-vibration signal containing two sinusoidal components with higher and lower frequencies that can be easily identified from the plot of the continuous wavelet coefficient in Fig. 3(b). In any time interval two frequencies are observed. It should be noted that the higher frequency can only be seen in small scale, i.e., lower portion of the plot, and vice versa for the lower frequency.

\section{DAMAGE DETECTION USING SIMULATION DATA FOR SIMPLE STRUCTURAL MODEL}

To demonstrate an application of the wavelet analysis for structural damage detection, a set of simulation data from a simple structural model are utilized. The structure is modeled as a single-degree-of-freedom (SDOF) mass-damper-spring system with multiple paralleled springs, as shown in Fig. 5. Each spring in the system is preassigned a threshold value, and a spring will break if the structural response exceeds its corresponding threshold value. In the case of accumulated damages, breakage of a spring is governed by the allowable number of cycles of the response based on fatigue testing. The springs are used to model stiffness of structural members such as columns, beams, and joints. Therefore, a broken spring may imply an occurrence of structural damage.

The governing differential equation of motion of the system in Fig. 5 is given by

$$
m \frac{d^{2}}{d t^{2}} x(t)+c \frac{d}{d t} x(t)+k(t) x(t)=f(t)
$$

where $m, c$, and $k=$ system mass, viscous damping coefficient,

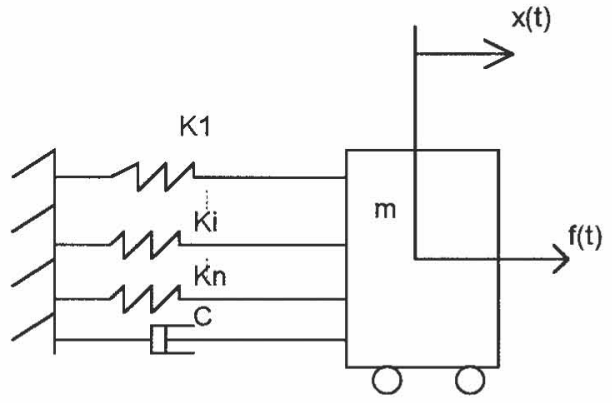

FIG. 5. Simple Structural Model with Multiple Paralleled Breakable Springs

and stiffness, respectively; $x(t)=$ displacement response; and $f(t)=$ external excitation. For the sake of simplicity, $f(t)$ is assumed to be a harmonic. The system stiffness $k(t)$ can be expressed by

$$
k(t)=\sum_{i=1}^{n} k_{i}(t)
$$

where $k_{i}(t)$ represents stiffness of the $i$ th spring in the system at time $t$. These springs are breakable due to different types of failure mechanism. For examples, if breakage of a spring is due to an excessive response, $k_{i}(t)$ can be defined as

$$
k_{i}(t)= \begin{cases}k_{i 0}, & \text { if } \operatorname{abs}\left(x\left(t^{\prime}\right)\right) \leq x_{i}^{*} \forall t^{\prime} \leq t \\ 0, & \text { otherwise }\end{cases}
$$

where $k_{i 0}$ and $x_{i}^{*}=$ initial stiffness and the threshold value of the $i$ th spring, respectively, and both are positive constants. If a spring is broken because of fatigue, $k_{i}(t)$ may be determined by

$$
k_{i}(t)= \begin{cases}k_{i 0}, & \text { if } N\left(t^{\prime}\right)<N_{i}^{*} \forall t^{\prime} \leq t \\ 0, & \text { otherwise }\end{cases}
$$

where $N(t)=$ total number of cycles of the response in the time interval $[0, t]$ and $N_{i}^{*}=$ allowable number of cycles for the $i$ th spring based on its fatigue testing. It is obvious that the system is nonlinear in general due to possible breakage of springs.

Fig. 6 plots the results for the above structural model to a harmonic excitation for the case where the damage is caused by an excessive response. For the sake of simplicity, only three springs were used for illustration. An analytical solution does not exist for nonlinear equation (12) with zero initial conditions due to the nonlinearity caused by possible breakage of springs. Therefore, the structural response was numerically 

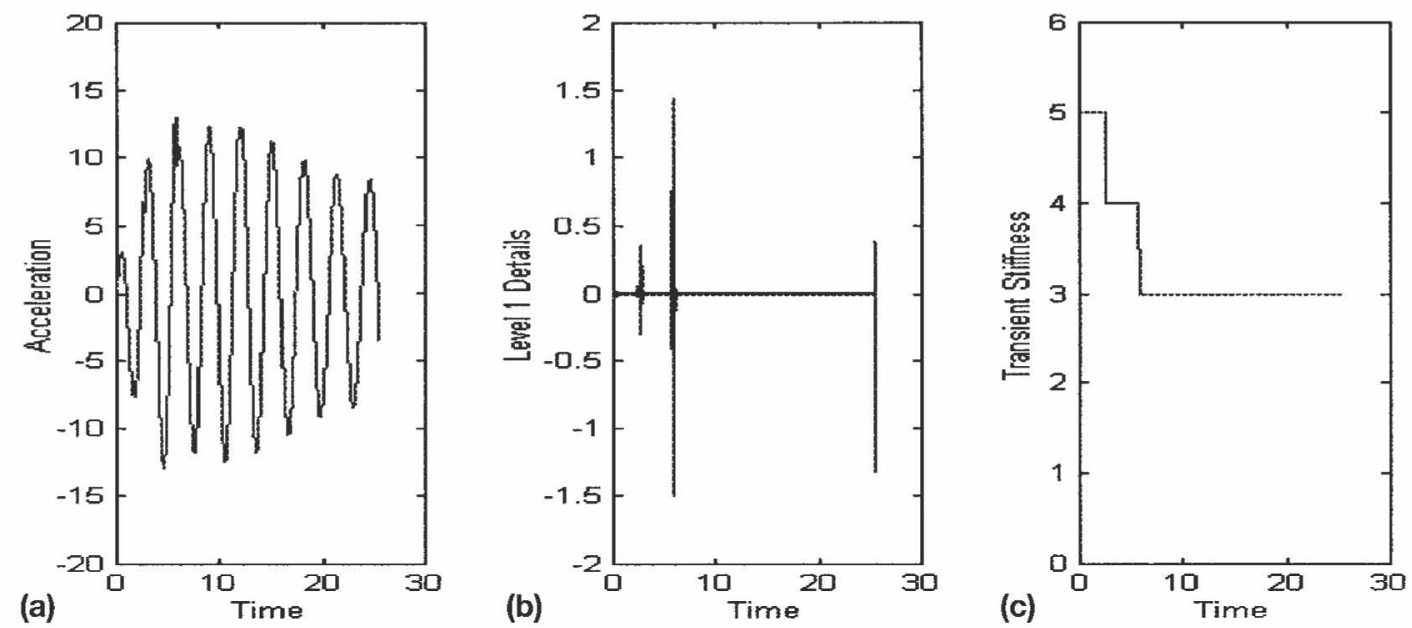

FIG. 6. Response of Simple Structural Model to Harmonic Input and Its Wavelet Analysis, Case 1-Damage due to Excessive Response: (a) Acceleration Response; (b) Level 1 Details in Wavelet Decomposition of Acceleration Response; (c) Transient Stiffness History of System
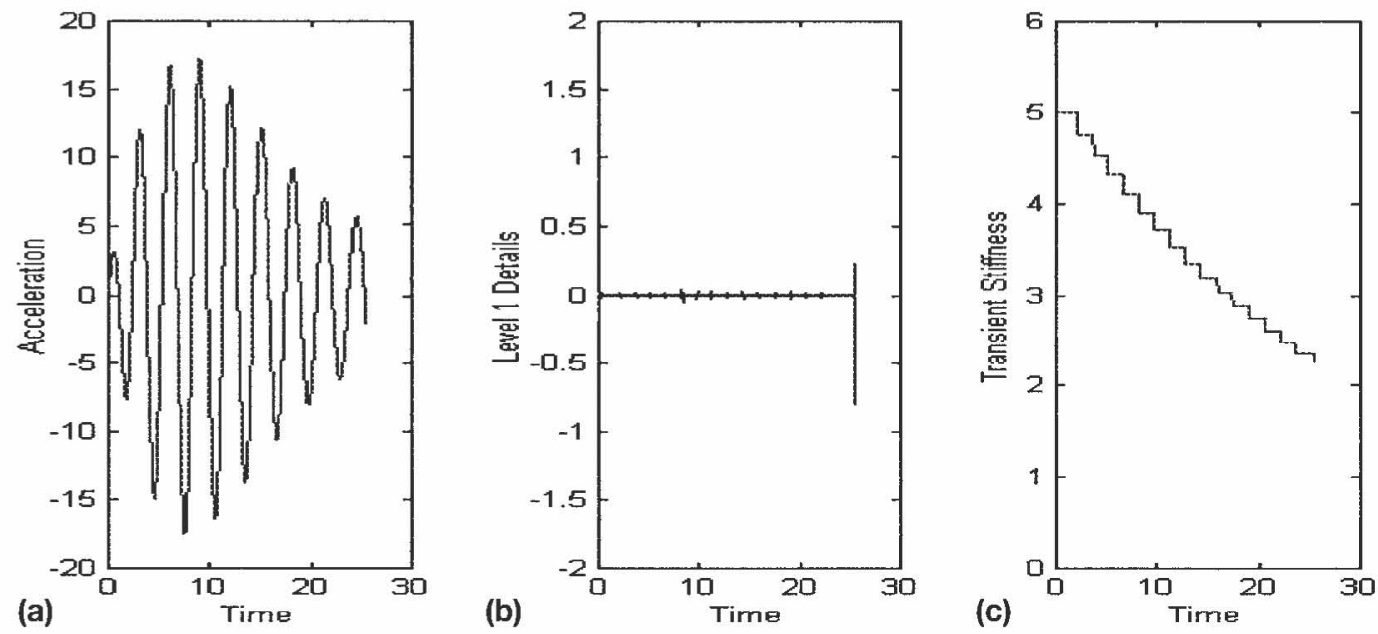

FIG. 7. Response of Simple Structural Model to Harmonic Input and Its Wavelet Analysis, Case 1-Damage due to Accumulative Fatigues: (a) Acceleration Response; (b) Level 1 Details in Wavelet Decomposition of Acceleration Response; (c) Transient Stiffness History of System
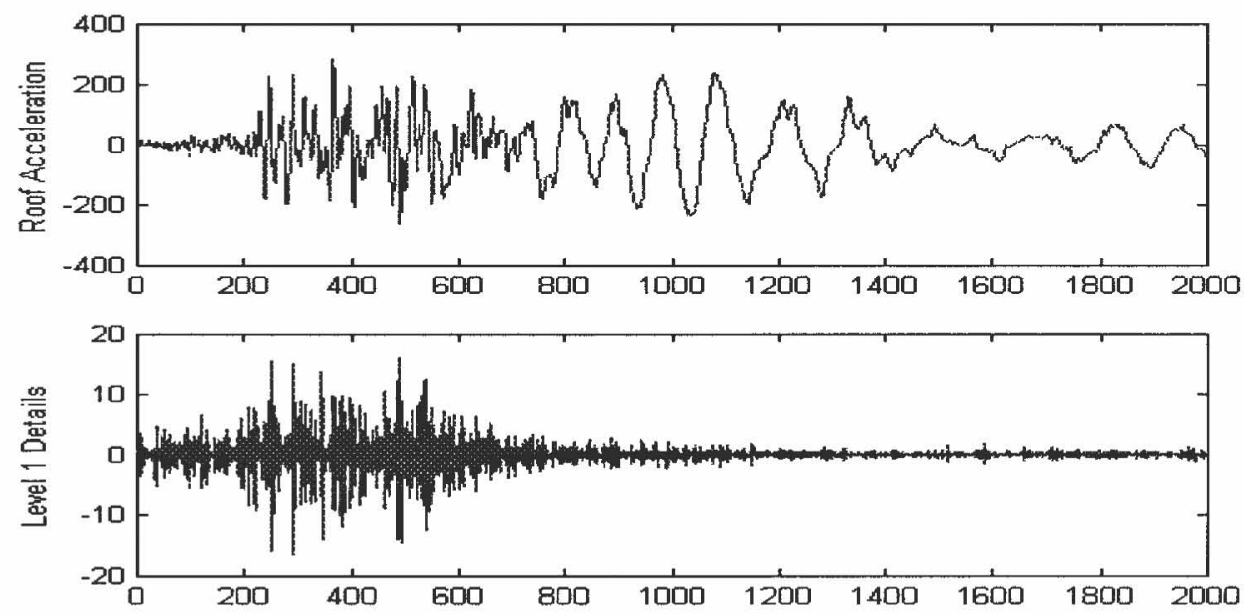

FIG. 8. Wavelet Analysis Results for Real Earthquake Response Data Recorded at Roof of Bank of California during 1971 San Fernando Earthquake

calculated by the fourth-order Runge-Kutta integration scheme. For the purpose of comparison, the transient stiffness history of the system was traced to see whether and when the springs break. While the stiffness history in Fig. 6(c) clearly indicated breakage of two springs, which results in stiffness degradation or loss at two different moments, the acceleration response curve apparently did not show signs of structural damage. However, results from wavelet analysis for the acceleration response data, as shown in Fig. 6(b), clearly show two spikes in the level 1 details of its wavelet decomposition, 
which indicate occurrence of structural damages. Level $j$ details are defined in (8). Compared with the stiffness history curve in Fig. 6(c), these two spikes accurately indicate the moments when the structural damage occurred. It should be noted that two spikes at the beginning and the end in the details curve are caused by truncation of the response data and should therefore be ignored.

Fig. 7 presents similar results for the case where the breakage of springs in the system is governed by their fatigue behavior, i.e., the total number of cycles of the system response $N(t)$. While there is not any sign in the acceleration response, as shown in Fig. 7(a), to indicate the structural damage, multiple spikes in the level 1 details of the acceleration signal in Fig. 7(b) imply progressive damage occurred. Again, the occurrences of damage are confirmed by the transient stiffness plot in Fig. 7(c).

\section{DAMAGE DETECTION USING RECORDED EARTHQUAKE RESPONSE DATA}

This section presents an example of application of the wavelet analysis for real earthquake response data recorded at the roof of the Bank of California building located at 15250 Ventura Boulevard in the city of Sherman Oaks, Calif., during the 1971 San Fernando earthquake. Its distance to the epicenter of the earthquake is approximately $14 \mathrm{mi}(22.53 \mathrm{~km})$. The building is a 12-story reinforced concrete moment-resisting structure. Plan dimension of the floors is $60 \times 161 \mathrm{ft}(18.29$ $\times 48.77 \mathrm{~m}$ ), except for the first story, which is $90 \times 161 \mathrm{ft}$ $(27.43 \times 49.07 \mathrm{~m})$. The building stands $159 \mathrm{ft}(48.46 \mathrm{~m})$ above the street level. During the San Fernando event, this structure suffered both structural and nonstructural damages. The structural damage consists mainly of cracks and spalling of columns and girder stubs.

Fig 8 illustrates the original acceleration response data recorded at the 7 th floor of the building and preliminary results from the wavelet analysis of the data. Characteristics of the spikes in the level 1 details of the DB4 wavelet decomposition seem in agreement with the field observation in the sense that the observed cracks and spalling of columns and stubs might occur subsequently during the earthquake event. The results show the great promise of wavelet approach for on-line SHM and postquake damage assessment. However, further experimental laboratory studies and field inspections on damaged structures are needed to justify the results and bring this approach into practical applications.

\section{EFFECTS OF NOISE INTENSITY AND DAMAGE SEVERITY}

To apply the wavelet approach to on-line health monitoring and damage detection many practical issues need to be addressed. For example, a recorded signal may be contaminated by noise due to various reasons. Also, the wavelet approach might be sensitive to the level of structural damage or stiffness loss. An attempt was made to investigate effects of the noise intensity and the damage level on detection of the structural damage using the wavelet approach. The results are presented in terms of a detectability map in Fig. 9.

The results in Fig. 9 were obtained by applying the wavelet approach to simulation data with added random noise and various levels of stiffness loss. The same structure system in Fig. 5 was used in this study. The noise was generated using a noise generator in MATLAB. Its intensity may be adjusted. For a given pair of noise intensity and damage level, wavelet analysis was employed for the response data to see if the damage could be detected based some preset criteria. The results were marked on a two-dimensional map with one axis representing the damage level and the other representing the noise intensity. A dark shaded cell indicates that damage is definitely detectable and a blank one indicates the opposite situation, i.e., strong noise and a small amount of damage make the damage detection impossible. A gray cell represents a questionable result based on the present criteria. Sometimes, a false detection might occur; if so, it is represented by a gray cell with the

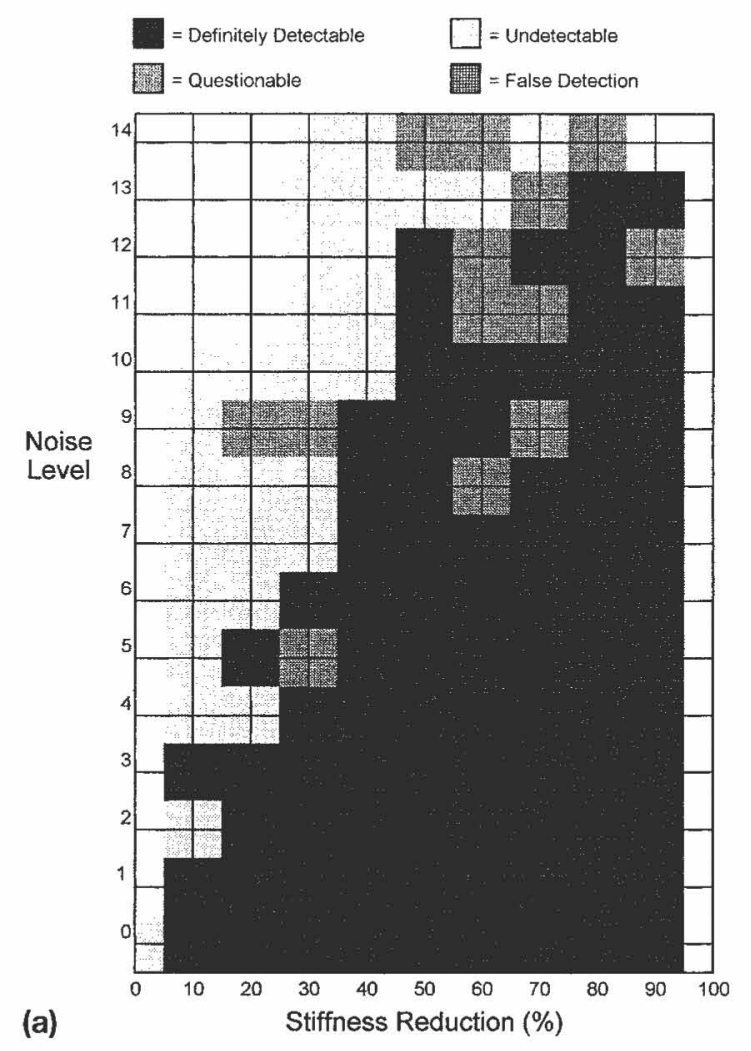

(a)

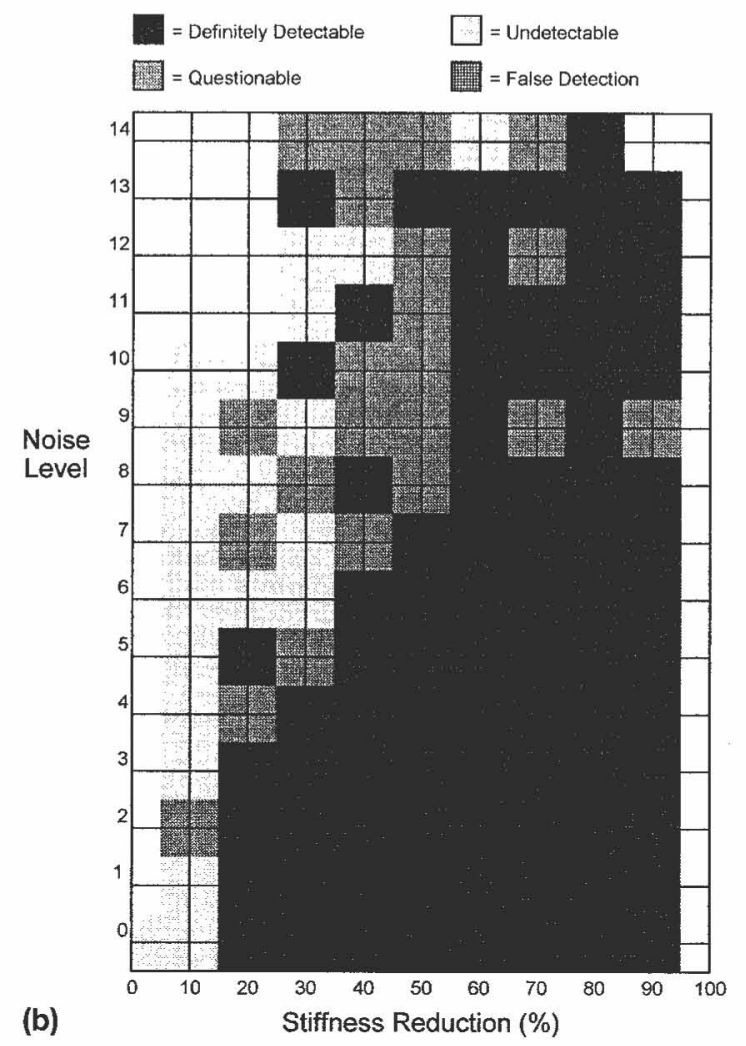

FIG. 9. Detectability Map for Effects of Noise Intensity and Damage Level on Damage Detection: (a) Results from Level 1 Details; (b) Results from Level 2 Details 
rectangular pattern. The results in Fig. 9 verify an intuitive experience that damage is more detectable for weaker noise and greater stiffness loss. While results based on both level 1 and level 2 details in the wavelet decomposition illustrate similar characteristics, damage seems more identifiable based on the level-1 details, as observed in Fig. 9(b). The detectability map provides a quantitative relationship between the noise intensity and the damage level for the damage detection.

\section{CONCLUSIONS}

This paper presents a wavelet-based approach for SHM and damage detection and demonstrates its applications using both numerical simulation data from a simple structural model with breakage springs and actual recorded data of the building response during an earthquake event. It shows that structural damage or a change in system stiffness may be detected by spikes in the details of the wavelet decomposition of the response data, and the locations of these spikes may accurately indicate the moments when the structural damage occurred. A detectability map is proposed to study the effects of noise contamination and damage severity on damage detection using the wavelet approach. It is a general conclusion that the damage is more detectable for a weaker noise and severer damage. The approach may be implemented both on-line and off-line, and therefore shows great promise for on-line health monitoring, integration with structural control, and postevent damage assessment.

\section{APPENDIX. REFERENCES}

Al-Khalidy, A., Noori, M., Hou, Z., Yamamoto, S., Masuda, A., and Sone, A. (1997a). "Health monitoring systems of linear structures using wavelet analysis." Proc., Int. Workshop on Struct. Health Monitoring: Current Status and Perspectives, Stanford University, Stanford, Calif., $164-175$.

Al-Khalidy, A., et al. (1997b). "A study of health monitoring systems of linear structures using wavelet analysis." ASME PVP Vol. 347, 49-58.

Benedetto, J. J., and Frazier, N. W., eds. (1993). Wavelets; mathematics and applications, CRC, Boca Raton, Fla.

Blanas, P., Wenger, M. P., Shuford, R. J., and Das-Gupta, D. K. (1997). "Active composite materials and damage monitoring." Proc., Int. Workshop on Struct. Health Monitoring: Current Status and Perspectives, Stanford University, Stanford, Calif., 199-207.

Champagne, V. K., Wechsler, G., and Pepi, M. (1998). "Metallurgical examination of a cracked output shaft from an army cargo helicopter." Proc., 52nd Meeting of the Soc. for Machinery Failure Prevention Technol.: Prognosis of Residual Life of Machinery and Struct. Haymarket, Va., 147-160.

Chang, F.-K., ed. (1997). Proc., Int. Workshop on Struct. Health Monitoring: Current Status and Perspectives, Stanford University, Stanford, Calif.

Chui, C. K. (1992). Introduction to wavelets, Academic, San Diego.

Daubechies, I., Mallat, S., and Willsky, A. S. eds. (1992). "Wavelet transforms and multiresolution signal analysis - special issue." IEEE Trans. Information Theory, 38(2).

Demetriou, M., and Hou, Z. (1999). "A comparison study of on-line fault diagnosis schemes." Proc., 13th Conf. on Engrg. Mech., ASCE, Reston, Va. (CD-ROM).

Doebling, S. W., Farrar, C. R., Prime, M. B., and Shevitz, D. W. (1996). "Damage identification and health monitoring of structural and me- chanical systems from changes in their vibration characteristics: a literature review." Rep. LA-13070-MS, Los Alamos National Laboratory, Los Alamos, N.M.

Essaway, M. A., Diwakar, S., Zein-Sabatto, S., and Garga, A. K. (1998). "Fault diagnosis of helicopter gearboxes using neuro-fuzzy techniques." Proc., 52nd Meeting of the Soc. for Machinery Failure Prevention Tech.: Prognosis of Residual Life of Machinery and Struct. Haymarket, Va., 293-302.

Ferlez, R. J., and Lang, D. C. (1998). "Gear-tooth fault detection and tracking using the wavelet transform." Proc., 52nd Meeting of the Soc. for Machinery Failure Prevention Technol.: Prognosis of Residual Life of Machinery and Struct., Haymarket, Va., 451-460.

Gurley, K., and Kareem, A. (1999). "Application of wavelet transform in earthquake, wind, and ocean engineering." Engrg. Struct., 21, 149167.

Hou, Z., and Noori, M. (1999). "Application of wavelet analysis for structural health monitoring." Proc., 2nd Int. Workshop on Struct. Health Monitoring, Stanford University, Stanford, Calif., 946-955.

Jiang, Z., Chonan, S., Kawashima, K., Muto, K., and Ichihara, W. (1997). "Condition monitoring of silicon-wafer slicer cutting crystal ingots." Proc., Int. Workshop on Struct. Health Monitoring: Current Status and Perspectives, Stanford University, Stanford, Calif., 624-635.

Kim, K. S., Kollar, L., and Springer, G. S. (1993). "A model of embedded fiber optic Fabry-Perot temperature and strain sensors." J. Compos. Mat., 27, 1618-1662.

Mallat, M. (1988). "A theory for multiresolution signal decomposition: The wavelet representation." IEEE Pattern Anal. and Machine Intelligence, 11(7), 674-659.

Masuda, A., Nakaoka, A., Sone, A., and Yamamoto, S. (1995). "Health monitoring system of structures based on orthonormal wavelet transform." Seismic Engrg., 312, 161-167.

Reigh, G. W., and Park, K. C. (1997). "Localized system identification and structural health monitoring from vibration test data." Proc., 1997 AIAA SDM Conf., American Institute of Aeronautics and Astronautics, Reston, Va.

Samuel, P. D., Pines, D. J., and Lewicki, D. G. (1998). "Fault detection in the OH-58A main transmission using the wavelet transform." Proc., 52nd Meeting of the Soc. for Machinery Failure Prevention Technol.: Prognosis of Residual Life of Machinery and Struct., Haymarket, Va., 323-336.

Sone, A., Yamamoto, S., Masuda, A., Nakaoka, A., and Ashino, R. (1995). "Estimation of cumulative damage of a building with hysteretic restoring force by using wavelet analysis of strong motion records." Japan Struct. Consr. Engrg., 476, 67-74.

Sone, A., and Yamamoto, S. (1997). Wavelet analysis: Its origination, development, and applications (in Japanese).

Staszewski, W. J., and Tomlinson, G. R. (1997). "Application of the wavelet transform to fault detection in spur gear." Mech. Sys. and Signal Processing, 8(3), 289-307.

Strang, G., and Nguyen, T. (1996). Wavelet and filter banks, WellesleyCambridge Press, Wellesley, Mass.

Tsou, P., and Shen, M. H. H. (1994). "Structural damage detection and identification using neural networks." AIAA J., 32(1), 176-183.

Vanik, M. W., and Beck, J. L. (1997). "A Bayesian probabilistic approach to structural health monitoring." Proc., Int. Workshop on Struct. Health Monitoring: Current Status and Perspectives, Stanford University, Stanford, Calif., 140-151.

Vincent, H. T., Hu, S.-L. J., and Hou, Z. (1999). "Damage detection using empirical mode decomposition method and a comparison with wavelet analysis." Proc., 2nd Int. Workshop on Struct. Health Monitoring, Stanford Univ., Stanford, Calif., 891-900.

Wang, W. J., and McFadden, P. D. (1995). "Application of orthogonal wavelets to early gear damage detection." Mech. Sys. and Signal Processing, 9(5), 497-507.

Wu, X., Ghabossi, J., and Garrett, J. H. (1992). "Use of neural networks in detection of structural damage." Comp. and Struct., 42(4), 649-659. 